\title{
Personalidade de atletas brasileiros de alto-rendimento: comparações entre os sexos masculino e feminino e correlação com nível de performance e tempo de treinamento
}

\author{
Maurício B. Filho ${ }^{1}$ \\ Luiz S. Ribeiro ${ }^{2}$ \\ Félix G. García ${ }^{3}$
}

\author{
${ }^{1}$ Universidade Federal de Juiz de Fora, Brasil \\ ${ }^{2}$ Infoteste do Brasil \\ ${ }^{3}$ Universidad de Las Palmas de Gran Canaria, Espanha
}

https://doi.org/10.5628/rpcd.05.01.31

\section{RESUMO}

Foi objetivo deste estudo avaliar as características da personalidade de atletas brasileiros de alto-rendimento, comparando as semelhanças e diferenças existentes entre indivíduos de ambos os sexos, assim como estabelecer correlações entre as variáveis psicológicas, níveis de performance e tempo de treinamento dos atletas. A amostra se constituiu de duzentos e nove atletas brasileiros (108 homens e 101 mulheres) de quatro modalidades esportivas (voleibol, basquete, judô e natação). Como instrumento para o estudo da personalidade, utilizou-se o Inventário de Personalidade de Freiburg (FPI-R). Foram encontradas diferenças estatisticamente significativas entre atletas homens e mulheres $(\mathrm{p}<0,05)$ em seis das doze variáveis do estudo: auto-realização, agressividade, inibição, irritabilidade, queixas físicas e emotividade. No entanto, esse comportamento não se repetiu quando a amostra foi subdividida por modalidades esportivas. As correlações encontradas entre as variáveis psicológicas do estudo e o tempo de treinamento $(-0,234<\mathrm{r}<$ $0,034)$ e o nível de performance $(-0,202<\mathrm{r}<0,043)$ dos atletas não foram estatisticamente significativas $(p>0,05)$. Em conclusão, os atletas brasileiros homens e mulheres de altorendimento, de uma maneira geral, apresentam diferenças nas características da personalidade. No entanto, quando os subgrupos foram separados e analisados de acordo com sua modalidade, não se observou uma quantidade significativa de diferenças entre atletas dos sexos masculino e feminino. As variáveis tempo de treinamento e nível de performance apresentaram correlações fracas com as variáveis de personalidade, porém sugere-se a realização de futuros estudos para aprofundar este tema.

Palavras-chave: personalidade, atletas brasileiros, gênero, performance, tempo de treinamento, FPI.

\begin{abstract}
Personality of Brazilian high-level athletes: comparison between men and women and correlation with performance level and training years.

This study aimed at (i) evaluating the personality characteristics of Brazilian high-level athletes, verifying similarities and differences among individuals from both gender and (ii) correlating psychological variables with performance level and training years. We sampled two hundred and nine athletes (108 men and 101 women) from four sports (volleyball, basketball, judo and swimming). The FPI-R (Freiburg Personality Inventory) was used to evaluate personality characteristics of the subjects. Statistical significant differences $(p<0,05)$ were found between Brazilian high-level male and female athletes in six out of twelve study variables: Life Satisfaction, Aggressiveness, Inhibition, Irritability, Physical Complaints and Emotionality. However, this tendency was not found when the sample was divided by the respective sports. The correlation found among the psychological variables and athletes training years $(-0,234<r<0,034)$ and performance level $(-0,202<r<0,043)$ was not statistically significant $(p>0,05)$. In conclusion: Men and women Brazilian high-level athletes, in general, presented differences in the personality characteristics. However, when divided and analyzed by their sports, it was not observed a significant difference between athletes from both sexes. Training years and performance level presented weak correlation when with the personality factors, but we suggest that more studies must be done to improve this topic.
\end{abstract}

Key Words: personality, Brazilian athletes, gender, performance, training years, FPI. 


\section{INTRODUÇÃo}

A relação entre personalidade e esporte tem sido uma das áreas de investigação mais exploradas pela Psicologia do Esporte. O volume de pesquisas e artigos publicados sobre o tema demonstra sua importância para pesquisadores, profissionais envolvidos no esporte e atletas $(9,22,23,27,29)$. A complexidade do tema aponta para a necessidade de aprofundamento de estudos em uma área que, até os dias de hoje, intriga aqueles que buscam explicações científicas para as diferenças de personalidade entre os atletas. Cox (4) e Auweele et al. (2), no entanto, indicavam para um decréscimo de interesse sobre o tema com a publicação de poucos artigos diretamente relacionados à personalidade. $\mathrm{O}$ que a princípio pode ser entendido como uma queda na importância dos estudos da personalidade no esporte pode ter outras explicações. Uma delas é a complexidade do tema. Muitas vezes, pesquisadores buscam respostas para dúvidas como "existe um perfil de personalidade dos campeões" e a insistência, juntamente com a ausência de explicações consistentes durante um longo período de tempo, pode ter causado um aparente desinteresse.

Entre essas razões, diversos são os autores que apontam lacunas no conhecimento entre a relação personalidade e esporte. Bara Filho et al. (3), Guillén e Castro (8), Junge et al. (11), Morris (13), O'Connor (14), Weinberg e Gould (29) e Williams e Reilly (30) enfatizam que o tema ainda está longe da perfeição, não havendo uma relação clara e precisa. Para os autores em questão, não existem evidências científicas de perfis de personalidade entre grupos de atletas de determinadas modalidades, bem como são escassos os estudos que auxiliem na determinação de diferenças de características psicológicas entre atletas jovens e atletas de alto-rendimento.

As diferenças entre homens e mulheres sempre foram e, ainda são, objeto de estudos nas mais diversas áreas do conhecimento e o esporte não se torna distinto. Características físicas, fisiológicas, no rendimento atlético e, também, nas variáveis psicológicas são estudadas exaustivamente para tentar explicar possíveis diferenças entre os sexos.

Alguns autores indicam que homens e mulheres atletas possuem perfis de personalidade semelhan- tes, apresentando poucas diferenças entre si (4, 9, $18,29)$. No entanto, outros estudiosos apontam diferentes características entre homens e mulheres atletas.

Para Pedersen (16), os atletas do sexo masculino diferenciam-se das mulheres atletas por serem mais ativos, apresentarem índices mais altos de agressividade, serem mais competitivos e controlados. As atletas diferenciam-se por serem mais organizadas e mais orientadas para um objetivo pré-determinado. No estudo de Cox e Liu (6) com atletas chineses e americanos das modalidades atletismo, basquete, vôlei e natação, as únicas diferenças constatadas, ao se avaliar o grupo de uma maneira geral, foram um maior nível de confiança e uma maior motivação entre os atletas homens, quando comparados às mulheres. Observa-se que as diferenças nas características entre atletas dos sexos masculino e feminino são mencionadas na literatura, porém não aparecem de forma constante, o que dificulta as conclusões a serem realizadas. Estudos mais sistematizados devem ser realizados para que esta lacuna no conhecimento seja preenchida. Por esta razão, diversos estudos recentes foram realizados comparando grupos de atletas homens e mulheres $(1,15)$ e, ainda assim, os resultados não se mostram consistentes. Os futuros estudos que objetivarem estudar as diferenças entre esses grupos devem também se preocupar em diferenciar atletas de não-atletas, pois os resultados obtidos com a população de atletas podem estar replicando as características gerais existentes entre homens e mulheres.

Dentro de um outro enfoque, atletas, treinadores e demais envolvidos no esporte competitivo sempre questionam a existência de características psicológicas específicas dos atletas de alto-rendimento em comparação àqueles de níveis inferiores. Muitas vezes, ouve-se afirmações que indicam o perfil de personalidade do grande atleta.

Quando considerados os níveis de performance, Cox (4) e Weinberg e Gould (29) sugerem que há indicações da relação da personalidade com a performance atlética, mas isso vem acompanhado por muitas imprecisões. O mesmo Cox (4) indica que existem características da personalidade, como estabilidade emocional, autodisciplina, capacidade mental de 
resistir ao esforço, baixa tensão, extroversão e independência que estão relacionadas com a performance. Peensgaard (17) completa, expondo que atletas de elite possuem uma maior persistência nos objetivos, dispondo de um maior nível de esforço para as tarefas determinadas.

Estudos recentes têm indicado diferenças entre atletas de níveis distintos de performance. Williams e Reilly (30) e Reilly et al. (21), avaliando talentos no futebol, concluíram que aqueles jogadores considerados mais talentosos possuíam uma maior autoconfiança, uma menor propensão a distúrbios da ansiedade e eram mais orientados à motivação pela tarefa e não pelo ego. Estudando atletas chineses, Cox et al. (5) concluíram que os atletas de alto-nível apresentavam habilidades psicológicas superiores em relação aos demais.

Num estudo com jogadores de futebol americano, Simpson e Newby (24) esclareceram que os atletas de melhor nível possuíam um melhor perfil de humor, com menores índices de raiva e hostilidade, indicando que a agressividade no esporte é diferente do conceito popular. Os atletas de melhor nível, supostamente, apresentam maiores índices de agressividade e isto foi comprovado por Rascle et al. (20) com jogadores de handebol, que também apresentaram orientação ao ego, ao contrário de estudos mencionados anteriormente.

Williams e Reilly (30) e Auweele et al. (2) concluem que os pesquisadores ainda necessitam identificar características específicas no perfil de personalidade relacionado com o sucesso no esporte, o que é corroborado por Morris (13), afirmando que não há pesquisas suficientes que diferenciem o atleta júnior do atleta de elite. Esses dados sugerem que este ainda é um campo vasto a ser explorado nos futuros estudos. Apesar de não se poder concluir satisfatoriamente, observa-se que existem características únicas dos atletas de alto-rendimento, no entanto isso deve ser melhor estudado e explorado em futuras pesquisas. Entre outras razões, este tópico de estudos dentro da personalidade vem crescendo nos últimos anos com a publicação de diversos artigos científicos (7, $12,19,25,28)$.

Pesquisas com populações de atletas de diferentes realidades sócio-culturais e econômicas, como as realiza- das com atletas chineses, devem ser feitas com atletas de outros países como o Brasil, pois a grande parte dos estudos realizou-se com norte-americanos e europeus. Isto posto, o presente estudo objetivou avaliar as características da personalidade de atletas brasileiros de alto-rendimento, verificando as semelhanças e diferenças existentes entre indivíduos de ambos os sexos, bem como estabelecendo correlações entre os distintos níveis de performance e o tempo de treinamento dos atletas.

\section{MATERIAL E MÉTODOS Caracterização da pesquisa}

Esta pesquisa caracteriza-se como descritiva, comparativa e correlacional. Descritiva, pois objetiva descrever sistematicamente características psicológicas da amostra de atletas brasileiros de ambos os sexos; comparativa devido a comparações a serem estabelecidas entre os sub-grupos de atletas masculino e feminino dessa amostra; e correlacional, pois pretende estabelecer uma correspondência entre as variáveis psicológicas, níveis de performance e tempo de treinamento dos atletas $(10,26)$.

\section{Amostra}

Um total de 209 (duzentos e nove) atletas de quatro modalidades esportivas (voleibol, basquetebol, judô e natação) constituiu a amostra, sendo 108 (cento e oito) atletas do sexo masculino e 101 (cento e um) do sexo feminino. As modalidades esportivas e suas respectivas características estão dispostas na Tabela 1. A idade média da amostra de atletas brasileiros é de $20,69 \pm 4,19$ anos e o tempo médio de treinamento é de $8,74 \pm 4,62$ anos. Para o cálculo do tempo de treinamento, estabeleceu-se o período entre o primeiro campeonato disputado pelo atleta nas categorias de base e o mês no qual foi realizada a coleta de dados. Quando divididos por grupos das modalidades esportivas de cada atleta, as variáveis idade e tempo de treinamento (em anos de prática) se comportam, respectivamente, da seguinte forma: natação $(21,25 \pm 4,82$ e $10,00 \pm 4,82$ anos), basquete $(25,63 \pm 5,70$ e $13,19 \pm 6,03$ anos), judô $(19,47 \pm 1,87$ e $8,98 \pm 3,54$ anos) e voleibol $(19,99 \pm 3,74$ e $6,82 \pm 3,73$ anos). 
Tabela 1: Características da amostra (número de indivíduos e idade média] de cada modalidade.

\begin{tabular}{lccccc}
\hline Modalidade & Natação & Basquete & Judô & Voleibol & Geral \\
\hline $\mathrm{N}$ [total] & 27 & 26 & 69 & 87 & 209 \\
$\mathrm{n}$ (Homens] & 11 & 26 & 31 & 40 & 108 \\
$\mathrm{n}$ & 16 & 0 & 38 & 47 & 101 \\
(mulheres] & & & & & \\
Idade & $21,25 \pm$ & $25,63 \pm$ & $19,47 \pm$ & $19,99 \pm$ & $20,69 \pm$ \\
média & 4,82 & 5,70 & 1,87 & 3,74 & 4,19 \\
(anos) & & & & & \\
Tempo de & $10,00 \pm$ & $13,19 \pm$ & $8,98 \pm$ & $6,82 \pm$ & $8,74 \pm$ \\
treinamento & 4,82 & 6,03 & 3,54 & 3,73 & 4,62 \\
\hline
\end{tabular}

O nível de performance estabelecido para os atletas participarem do estudo constituiu-se no fato deles estarem no mínimo competindo nos campeonatos nacionais adultos ou ligas nacionais de seus respectivos esportes (2). Para esta variável, estabeleceu-se uma escala de 1 (um) a 10 (dez), sendo 1 (um) para aquele atleta participante somente do campeonato nacional e 10 (dez) para aquele tenha disputado um Campeonato Mundial adulto e/ou os Jogos Olímpicos.

Em relação a esta variável, detectou-se que $100 \%$ dos atletas participavam dos campeonatos nacionais em suas respectivas modalidades, $85,3 \%$ já haviam representado a seleção brasileira em alguma idade (juvenil ou adulta) e que $29,1 \%$ da amostra estava participando, ou partipou em algum momento de sua carreira desportiva, das equipes principais do Brasil em campeonatos como Sul-americanos, Panamericanos, Mundiais e Jogos Olímpicos.

\section{Instrumento da pesquisa}

O instrumento utilizado foi o Inventário de Personalidade de Freiburg (FPI-R), na sua versão revista, contendo 138 questões com possibilidades de respostas entre concordo e não concordo, sendo aplicado uma única vez. As seguintes variáveis foram estudadas: Auto-realização, Espírito Humanitário, Empenho Laboral, Inibição, Irritabilidade, Agressividade, Fatigabilidade, Queixas Físicas, Preocupação com a Saúde, Sinceridade, Extroversão, Emotividade. O instrumento foi respondido de maneira voluntária em momentos antes ou após o treinamento ou competições desses atletas.

O FPI caracteriza-se como um inventário de personalidade que descreve de forma objetiva a manifestação de importantes dimensões da personalidade, permitindo uma comparação interindividual. Isso vem a classificá-lo como um importante instrumento para pesquisas que objetivem comparar diferentes grupos de indivíduos. Além do FPI, os atletas preencheram um questionário geral contendo informações como sexo, idade, tempo de treinamento desportivo e nível de performance.

\section{Analise estatística}

Para a análise da existência de características da personalidade entre atletas de ambos os sexos, utilizouse inicialmente a estatística descritiva (média e desvio-padrão) para o comportamento de cada variável do estudo. Posteriormente, aplicou-se um teste " $t$ " de Student para verificar a existência de diferenças estatisticamente significativas entre as médias dos sub-grupos (masculino e feminino). Para a comparação entre atletas do mesmo sexo e diferentes modalidades, bem como sexos e modalidades diferentes, utilizou-se uma ANOVA one-way com Post-Hoc de Sheffé para analisar as diferenças entre as médias das variáveis. O índice de correlação de Pearson foi utilizado para o cálculo das relações entre as variáveis psicológicas do estudo com as variáveis nível de performance e tempo de treinamento..

\section{RESULTADOS}

Na Tabela 2, pode-se observar as médias e desviospadrão das variáveis psicológicas dos dois grupos, masculino e feminino, respectivamente. Nesse primeiro momento, os atletas foram separados somente por sexo, desconsiderando suas modalidades esportivas. 
Tabela 2: Estatística descritiva - média (desvio-padrão) em pontos - das variáveis psicológicas do estudo do grupo geral e separadamente de atletas masculino e feminino e teste " $t$ " de Student para diferença entre médias.

\begin{tabular}{|c|c|c|c|c|c|}
\hline Variável/Modalidade & Geral & Mulheres & Homens & & \\
\hline & $X \pm D P$ & $X \pm D P$ & $\mathrm{X} \pm \mathrm{DP}$ & $" \mathrm{t} "$ & $\mathbf{P}$ \\
\hline Auto-realização & $7,78 \pm 2,23$ & $7,42 \pm 2,41$ & $8,11 \pm 2,01$ & $-2,240$ & $0,026^{*}$ \\
\hline Espírito Humanitário & $8,04 \pm 1,86$ & $8,16 \pm 1,96$ & $7,92 \pm 1,77$ & 0,900 & 0,369 \\
\hline Empenho Laboral & $8,64 \pm 1,91$ & $8,60 \pm 1,89$ & $8,67 \pm 1,94$ & $-0,236$ & 0,813 \\
\hline Inibição & $4,37 \pm 2,28$ & $4,69 \pm 2,34$ & $4,07 \pm 2,19$ & 1,975 & $0,050^{*}$ \\
\hline Irritabilidade & $5,03 \pm 2,55$ & $5,72 \pm 2,46$ & $4,39 \pm 2,47$ & 3,906 & $0,000^{* * *}$ \\
\hline Agressividade & $4,01 \pm 2,36$ & $3,64 \pm 2,17$ & $4,35 \pm 2,48$ & $-2,190$ & $0,030^{*}$ \\
\hline Fatigabilidade & $5,15 \pm 2,74$ & $5,35 \pm 2,62$ & $4,96 \pm 2,84$ & 1,112 & 0,313 \\
\hline Queixas Físicas & $3,03 \pm 2,17$ & $3,80 \pm 2,32$ & $2,31 \pm 1,74$ & 5,267 & $0,000^{* * *}$ \\
\hline Preocup com saúde & $6,72 \pm 2,49$ & $6,98 \pm 2,47$ & $6,47 \pm 2,49$ & 1,480 & 0,140 \\
\hline Sinceridade & $6,62 \pm 2,34$ & $6,46 \pm 2,48$ & $6,77 \pm 2,21$ & $-0,936$ & 0,351 \\
\hline Extroversão & $10,06 \pm 2,54$ & $9,82 \pm 2,65$ & $10,28 \pm 2,42$ & $-1,299$ & 0,195 \\
\hline Emotividade & $6,20 \pm 2,92$ & $6,77 \pm 3,25$ & $5,68 \pm 2,48$ & 2,753 & $0,006^{* *}$ \\
\hline
\end{tabular}

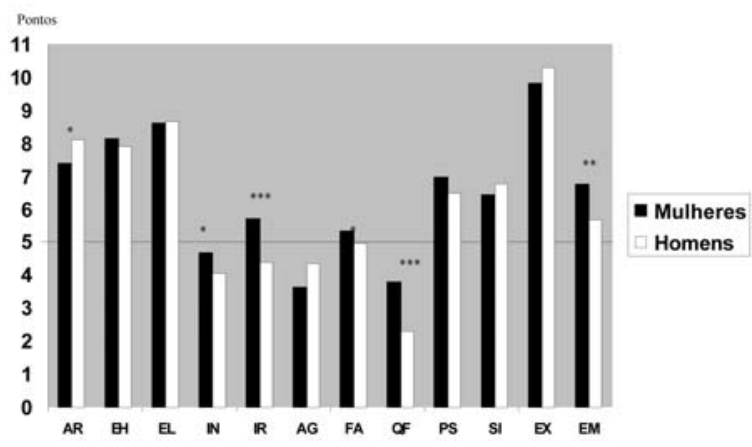

Figura 1: Disposição das médias das variáveis do FPI entre atletas homens e mulheres. ( ${ }^{*} p<0,05,{ }^{* *} p<0,01,{ }^{* * *} p<0,001$ ).

Legenda: AR-Auto-realização; $\varepsilon H$ - Espírito Humanitário; $E$ L- Empenho Laboral; IN-Inibição; IR- Irritabilidade; AG-Agressividade; FA- Fatigabilidade; QF- Queixas Físicas; PS- Preocupação com a Saúde; SI- Sinceridade; EXExtroversão; EM- Emotividade.

Inicialmente, torna-se claro na Tabela 2 e, também, na Figura 1 a existência de diferenças de médias entre atletas do sexo masculino e feminino nas variáveis psicológicas dos estudos. As variáveis que apresentaram maiores diferenças nas médias foram Queixas Físicas (3,80 e 2,31 pontos para mulheres e homens, respectivamente), irritabilidade (5,72 e 4,39 pontos) e emotividade (6,77 e 5,68 pontos), enquanto as que variaram menos foram Empenho Laboral (8,60 e 8,67 pontos), Espírito Humanitário $(8,16$ e 7,92 pontos) e sinceridade $(6,46$ e 6,77 pon- tos). No entanto, essas diferenças necessitam ser testadas estatisticamente para a verificação de diferenças significativas entre elas. Para isto, foi aplicado o teste " $t$ " de Student para amostras independentes (Figura 1 e Tabela 2).

Pode-se observar na Tabela 2 e na Figura 1 que foram encontradas diferenças estatisticamente significativas $(\mathrm{p}<0,05)$ em seis das doze variáveis do estudo. Analisando-as, verifica-se que os atletas do sexo masculino apresantam maiores escores nas variáveis auto-realização $(p<0,05)$ e agressividade $(p<0,05)$, caracterizando-os de uma maneira geral como mais confiantes, com uma maior satisfação geral, mais bem humorados, com maior conduta agressiva que as mulheres. Estas, no entanto, demonstram maiores escores que os homens nas variáveis inibição $(p=0,05)$, irritabilidade $(p<0,001)$, queixas físicas $(p<0,001)$ e emotividade $(p<0,01)$, o que as diferencia como mais tímidas e com certa dificuldade de expressão em público, com mais reações psicossomáticas, mais sensíveis e emocionalmente mais lábeis que os homens. Observa-se, de maneira geral, diferenças existentes entre esses dois grupos.

Para prosseguir aprofundando a análise, realizou-se uma análise descritiva e de variância (ANOVA) para comparar os grupos de homens e mulheres da mesma modalidade e de modalidades diferentes, respectivamente. A Tabela 3 demonstra as médias e os desvios-padrão de cada subgrupo. 
Tabela 3: Estatística descritiva - média \pm desvio-padrão em pontos - das variáveis psicológicas do estudo dos subgrupos de atletas masculino $(M)$ e feminino $(F)$ das diferentes modalidades.

\begin{tabular}{lccccccc}
\hline $\begin{array}{l}\text { Variável/ } \\
\text { Modalidade }\end{array}$ & $\begin{array}{c}\text { Vôlei } \\
\text { [F] }\end{array}$ & $\begin{array}{c}\text { Vôlei } \\
\text { (M) }\end{array}$ & $\begin{array}{c}\text { Basquete } \\
\text { (M) }\end{array}$ & $\begin{array}{c}\text { Judô } \\
\text { [F] }\end{array}$ & $\begin{array}{c}\text { Judô } \\
\text { (M) }\end{array}$ & $\begin{array}{c}\text { Natação } \\
\text { (F) }\end{array}$ & $\begin{array}{c}\text { Natação } \\
\text { (M) }\end{array}$ \\
\hline Auto-realização & $8,34 \pm 2,13$ & $8,65 \pm 1,85$ & $7,96 \pm 2,22$ & $6,20 \pm 2,57$ & $7,68 \pm 1,81$ & $7,44 \pm 1,62$ & $7,72 \pm 2,37$ \\
Esp. Humanitário & $8,40 \pm 1,80$ & $8,80 \pm 1,50$ & $7,65 \pm 1,62$ & $8,11 \pm 2,09$ & $7,45 \pm 1,70$ & $7,56 \pm 2,06$ & $6,73 \pm 2,00$ \\
Empenho Laboral & $8,81 \pm 1,82$ & $9,05 \pm 1,45$ & $8,54 \pm 1,90$ & $8,24 \pm 2,02$ & $8,19 \pm 2,39$ & $8,87 \pm 1,75$ & $8,91 \pm 2,12$ \\
Inibição & $4,42 \pm 1,89$ & $3,87 \pm 1,98$ & $4,00 \pm 2,24$ & $5,02 \pm 2,92$ & $4,09 \pm 2,31$ & $4,56 \pm 1,96$ & $4,91 \pm 2,55$ \\
Irritabilidade & $5,42 \pm 2,17$ & $3,70 \pm 1,95$ & $4,73 \pm 3,10$ & $5,97 \pm 2,73$ & $4,71 \pm 2,24$ & $6,00 \pm 2,66$ & $5,18 \pm 2,86$ \\
Agressividade & $3,47 \pm 1,78$ & $3,97 \pm 2,07$ & $3,65 \pm 2,61$ & $3,92 \pm 2,72$ & $5,03 \pm 2,66$ & $3,50 \pm 1,71$ & $5,45 \pm 2,45$ \\
Fatigabilidade & $4,83 \pm 2,40$ & $4,05 \pm 2,18$ & $5,27 \pm 2,84$ & $5,97 \pm 2,83$ & $5,68 \pm 3,41$ & $5,37 \pm 2,53$ & $5,54 \pm 2,73$ \\
Oueixas Físicas & $3,89 \pm 2,11$ & $2,10 \pm 1,48$ & $2,11 \pm 1,68$ & $4,16 \pm 2,54$ & $2,35 \pm 1,83$ & $2,69 \pm 2,15$ & $3,45 \pm 2,21$ \\
Preocup com Saúde & $7,45 \pm 1,94$ & $7,03 \pm 2,70$ & $5,73 \pm 1,97$ & $6,29 \pm 2,81$ & $6,26 \pm 2,73$ & $7,25 \pm 2,77$ & $6,82 \pm 1,66$ \\
Sinceridade & $5,83 \pm 2,35$ & $5,97 \pm 2,08$ & $6,31 \pm 2,09$ & $7,26 \pm 2,33$ & $7,77 \pm 2,09$ & $6,44 \pm 2,76$ & $7,91 \pm 1,87$ \\
Extroversão & $10,40 \pm 2,33$ & $10,40 \pm 2,68$ & $9,69 \pm 2,54$ & $9,29 \pm 2,85$ & $10,97 \pm 1,72$ & $9,37 \pm 2,87$ & $9,27 \pm 2,49$ \\
Emotividade & $6,23 \pm 2,93$ & $5,25 \pm 2,13$ & $5,35 \pm 2,98$ & $7,42 \pm 3,51$ & $6,59 \pm 2,41$ & $6,81 \pm 3,41$ & $5,54 \pm 2,29$ \\
\hline
\end{tabular}

A ANOVA das diferenças encontradas entre as médias da Tabela 3 demonstrou que há diferenças estatisticamente significativas ns variáveis Auto-realização $(p<0,001)$, Espírito Humanitário $(p<0,01)$, Irritabilidade $(p<0,01)$, Queixas Físicas $(\mathrm{p}<0,001)$ e Sinceridade $(\mathrm{p}<0,01)$ quando os sub-grupos são analisados separadamente. Para verificar essas diferenças demonstradas na ANOVA, utilizou-se o Teste Post-Hoc de Sheffé. Os resultados estão dispostos na Tabela 4 para que sejam analisados separadamente, a fim de um melhor entendimento.

Tabela 4: Post-Hoc de Sheffé entre homens e mulheres de diferentes modalidades.

\begin{tabular}{ccc}
\hline & & Mulheres \\
\hline Homens & Judô & Voleibol \\
\hline Voleibol & Auto-realização $(p=0,001)$ & Oueixas Físicas $(p=0,011)$ \\
& Irritabilidade $(p=0,013)$ & \\
Oueixas Físicas $(p=0,003)$ & Sinceridade $(p=0,033)$ \\
Judô & Queixas Físicas $(p=0,017)$ & Oueixas Físicas $(p=0,047)$ \\
\hline
\end{tabular}

$$
\left({ }^{*} p<0,05,{ }^{* *} p<0,01,{ }^{* * *} p<0,001\right)
$$

Na Tabela 4, observa-se diferenças significativas entre homens do voleibol e mulheres do judô nas variáveis Auto-realização $(\mathrm{p}<0,01)$, Irritabilidade $(\mathrm{p}<0,05)$ e Queixas Físicas $(p<0,01)$, constituindo-se nos grupos que mais quantidade diferenças apresentaram. As judocas também se diferenciaram dos jogadores de basquete na variável Queixas Físicas $(\mathrm{p}<0,05)$. Outras diferenças significativas foram encontradas entre as atletas mulheres de voleibol na variável Queixas Físicas, respectivamente, com homens desta mesma modalidade $(\mathrm{p}<0,05)$ e com jogadores de basquete $(\mathrm{p}<0,05)$, assim como na variável
Sinceridade $(\mathrm{p}<0,05)$ com os judocas.

A partir dos resultados apresentados na Tabela 4, principalmente, deve-se discutir que existem diferenças entre atletas de alto-rendimento de ambos os sexos, no entanto a quantidade de diferenças encontradas não pode ser considerada significativa, devido à existência de sete sub-grupos (vôlei, natação e judô, masculino e feminino e o basquete, somente masculino, combinados com as doze variáveis do estudo). Ainda assim, os resultados apresentados indicam haver um maior número de diferenças na comparação de esportes de contato físico direto (ex. judô) 
com aqueles indiretos (ex. vôlei), o que pode indicar uma direção para as diferenças psicológicas no perfil dos atletas.

Outro fator é a pouca quantidade de diferenças entre homens e mulheres da mesma modalidade esportiva, fato este que aponta para características semelhantes entre atletas de uma mesma modalidade esportiva. Deve-se salientar também que as diferenças entre indivíduos do mesmo sexo e modalidades esportivas diferentes foi muito pequena, com a maior parte ocorrendo entre homens e mulheres.

Para avaliar o grau de relação das variáveis psicológicas estudadas com o nível de performance dos atletas, bem como com o tempo de treinamento submetido até então, utilizou-se o índice de correlação de Pearson. Os resultados indicaram a inexistência de correlações altas entre as variáveis psicológicas do estudo e o tempo de treinamento $(-0,234<\mathrm{r}<$ $0,034)$ e o nível de performance $(-0,202<\mathrm{r}<0,043)$ dos atletas. Esses dados podem estar indicando uma estabilidade das variáveis de personalidade, mesmo quando confrontadas com outras, assim essas tendem a se manter estáveis durante vários anos.

A pequena relação entre essas variáveis estudadas reforça a teoria da existência de um traço de personalidade que pouco se modifica no decorrer dos anos, enfraquecendo a idéia da modificação da personalidade com a prática esportiva. No entanto, isso só poderá ser comprovado através de uma pesquisa longitudinal e não de uma maneira transversal como o presente estudo foi realizado.

\section{CONSIDERAÇÕES FINAIS}

A necessidade de se caracterizar e diferenciar grupos distintos ainda motiva muitos pesquisadores das mais amplas áreas do conhecimento científico. $\mathrm{E}$ essa constatação aplica-se diretamente às Ciências do Esporte e à Psicologia Esportiva. Preencher lacunas do conhecimento, como as ainda existentes nos estudos de personalidade de atletas, deve se tornar um fomentador e motivador na produção do conhecimento, por mais complexo que seja o tema.

Diferenciar indivíduos dos sexos masculino e feminino e, também, pessoas com distintos níveis de performance têm sido alguns desses objetos de estudo em diversas áreas, refletindo também na Psicologia do Esporte nas suas pesquisas sobre características da personalidade.
Dentro dos objetivos propostos no presente estudo, pode-se observar que os atletas brasileiros de altorendimento apresentam diferenças quando separados quanto ao sexo. Atletas homens e mulheres diferenciaram nas variáveis auto-realização, agressividade, inibição, irritabilidade, queixas físicas e emotividade, quando separados de uma maneira geral sem considerar suas respectivas modalidades.

No entanto, quando os subgrupos de atletas brasileiros, homens e mulheres, foram separados e analisados de acordo com sua modalidade, não observou-se uma quantidade significativa de diferenças entre atletas dos sexos masculino e feminino. Apenas em algumas variáveis (auto-realização, queixas físicas, sinceridade, irritabilidade) e entre alguns subgrupos foram encontradas diferenças estatisticamente significativas. Mas apesar dessa pequena quantidade de diferenças entre atletas do sexo masculino e feminino, algumas considerações importantes que poderão acrescentar ao conhecimento científico da personalidade no esporte estão presentes neste estudo.

As diferenças mais significativas ocorreram entre indivíduos de diferentes sexos e entre modalidades esportivas de contato físico direto (Ex. Judô) com aquelas que não tem contato físico direto (Ex. Vôlei). Esse realmente torna-se um referencial do presente estudo e aponta para futuras pesquisas sobre o assunto. Há indícios que características psicológicas não determinam a escolha de uma determinada modalidade, mas podem indicar o tipo de esporte a ser escolhido pelo individuo como forma sistematizada de treinamento.

Outro ponto importante ocorre quando os subgrupos são analisados e comparados conjuntamente. Observa-se muito poucas diferenças entre indivíduos de sexos diferentes e praticantes da mesma modalidade esportiva. Essa constatação pode indicar a existência de características psicológicas semelhantes entre atletas de um determinado esporte.

Os resultados encontrados no presente estudo corroboram intensamente com os estudos apresentados por Cox (4), Cox e Liu (6), Hernandez-Ardieta et al. (9) e Weinberg e Gould (29), que já indicavam que homens e mulheres atletas possuem perfis de personalidade semelhantes.

Há também uma semelhança com os achados de Pedersen (16), quanto à variável agressividade. Em 
seu estudo, os atletas homens apresentaram índices mais altos de agressividade que as mulheres.

Resumidamente, observa-se que a maior parte das diferenças significativas das variáveis psicológicas ocorreu entre homens e mulheres, entre modalidades distintas e entre modalidades de contato direto e indireto. Apesar das diferenças entre sexos existirem, não são tão grandes, demonstrando que pode existir um perfil de personalidade de atletas de altorendimento. Esse dado, no entanto, só poderá ser corroborado quando este grupo de atletas for comparado com um de não atletas.

De uma maneira contrária ao conhecimento prévio sobre o assunto, não foram encontradas altas correlações entre as variáveis tempo de treinamento e nível de performance, quando comparadas com as variáveis psicológicas abordadas neste estudo. Este resultado deve ser entendido, no entanto, pelo fato da amostra de atletas brasileiros ser de certa forma homogênea por se tratar de atletas de alto-rendimento, com estes apresentando poucas diferenças no seu nível de performance.

Comparando com a produção científica anterior sobre o tema, Cox (4) e Weinberg e Gould (29) já sugeriam que a relação da personalidade com a performance atlética estava cercada de imprecisões. No entanto, Cox (4), Cox et al. (5), Peensgaard (17), Simpson e Newby (24), Reilly et al. (21) e Williams e Reilly (30) apontaram diferenças entre atletas de diferentes níveis de performance.

Este fato não foi comprovado pelo presente estudo. No entanto deve-se ressaltar que a amostra do presente estudo era toda composta por atletas de altorendimento, o que pode ter influenciado no resultado, devido à pequena diferença existente entre seus níveis de rendimento.

Desta forma, o presente estudo corrobora com Williams e Reilly (30) e Morris (13) quanto à necessidade de identificar características específicas no perfil de personalidade relacionado com o sucesso no esporte.

Com toda esta gama de informações, caminhos futuros para as pesquisas sobre o tema personalidade e esporte podem ser sugeridos para uma maior e melhor compreensão de um tema tão complexo. Para que realmente constate-se a existência de um perfil de personalidade do atleta de alto-rendimento, essa população e seus respectivos subgrupos devem ser comparados com uma população de não-atletas, para que as diferenças inter e intra-grupos sejam melhor compreendidas.

Quanto à avaliação da relação de variáveis como tempo de treinamento e nível de performance e as variáveis psicológicas, sugere-se a realização, tanto de estudos com atletas e praticantes de diferentes níveis de performance, como de pesquisas longitudinais para verificar possíveis evoluções nas características psicológicas dos atletas com o decorrer de toda sua carreira desportiva.

Para finalizar, deve-se continuar a incentivar estudos sobre a personalidade de atletas em regiões que ainda necessitam ser mais exploradas como Américas do Sul e Central, África e Ásia, para que todas as variáveis intervenientes no processo de formação dos atletas sejam exploradas.

\section{AGRADECIMENTOS}

A todos os atletas brasileiros que participaram da coleta de dados voluntariamente e aos membros das comissões técnicas dos seguintes desportos que possibitaram a realização deste estudo: Voleibol (Professores Bernardo Rezende, Marco Aurélio Cunha, Antonio Rizola, Marcos Lerbach, Percy Oncken, Luiziomar Moura e Wadson Lima, e aos supervisores Hélcio Nunan e Jorge de Barros Confederação Brasileira de Voleibol); Natação (Professores João Carlos Barros e Ricardo Moura Confederação Brasileira de Desportos Aquáticos); Basquete (Professores André Guimarães e Roberto Ribeiro de Almeida); e Judô (Professores Josúe Morrison Moraes e Paulo Wanderley - Confederação Brasileira de Judô).

Parte desse artigo foi apresentado no $10^{\circ}$ Congresso de Ciências do Desporto e de Educação Física dos Países de Língua Portuguesa, com resumo publicado na Revista Portuguesa de Ciências do Desporto - v. $4, \mathrm{n}^{\circ} 2$ (suplemento), p. 146.

\section{CORRESPONDÊNCIA}

\section{Maurício Gattás Bara Filho}

Rua São Sebastião, 1295/901

Juiz de Fora - MG

36015-410 Brasil

mgbara@terra.com.br 


\section{REFERÊNCIAS BIBLIOGRÁFICAS}

1. Anshel MH, Eom HJ (2003). Exploring the dimensions of perfectionism in sport. Int J Sport Psychol 34(3): 255-271.

2. Auweele YV, Nys K, Rzewnicki, R, Mele V (2001). Personality and athlete. In: R. Singer R, HA Haussenblas, CM Janelle (Org). Handbook of Sport Psychology. New York: John Wiley \& Sons, 239-268.

3. Bara Filho MG, Ribeiro LS, Guillén F (in press). La personalidad de deportistas brasileños de alto nivel: comparación entre diferentes modalidades deportivas. Cuad Psicol Deporte.

4. Cox RH (1994). Sport Psychology: concepts and applications. Dubuque: Brown \& Benchmark.

5. Cox RH, Liu Z, Qiu Y (1996). Psychological skills of elite Chinese athletes. J Sport Psy 27: 123-132.

6. Cox RH, Liu Z (1993). Psychological skills: a cross cultural investigation. Int J Sport Psychol 24: 326-340.

7. Gould D, Dieffenbach K, Moffett A (2002). Psychological characteristics and their development in Olympic champions. J App Sport Psychol 14(3): 172-204.

8. Guillén F, Castro JJ (1994). Comparación de la personalidad en deportistas y no deportistas, utilizando como instrumento el EPQ-A de Eysenk. Rev Psicol Deporte 5: 5-14.

9. Hernandez-Ardieta IP, Lopez JC, Dolores M, Ruiz, EJG (2002). Personalidad, diferencias individuales y ejecución deportiva. In: AO Zafra, HJ Ruiz, GN Garcia (Coords). Manual de Psicología del Deporte. Murcia: DM, 105-123.

10. Isaac S, Michael WB (1993). Handbook in research and evaluation. San Diego: Edits.

11. Junge A, Dvorak J, Rosch D, Graf-Baumann T, Chomiak J, Peterson L (2000) Psychological and Sport-Specific Characteristics of Football Players. Am J Sports Med 28(5): S22-S28.

12. Kitsantas A, Zimmerman B (2002). Comparing self-regulatory processes among novice, non-expert, and expert volleyball players: A microanalytic study. J App Sport Psychol 14(2): 91-105.

13. Morris T (2000). Psychological characteristics and talent identification in soccer. J Sports Sci 18: 715-726.

14. O’Connor PJ (1996). Aspectos psicológicos del rendimiento de resistencia. In: RJ Shephard, PO Astrand (Org). La Resistencia en el deporte. Barcelona: Paidotribo, 149-156.

15. Pappalardo A, Festuccia E, Pesce C (2001). Differenze di genere nel profilo internazionale di atleti praticanti pallavolo. Movimento 17(3): 27-30.

16. Pedersen DM (1997). Perceived traits of male and female athletes. Percept Mot Skills 85: 547-550.

17. Peensgard AM (1999). The dynamics of motivation and perceptions of control when competing in the Olympic Games. Percept Mot Skills 89: 116-125.

18. Petterson TW, Wendt DT. (1974). Personality characteristics of women in intercollegiate competition. Percept Mot Skills 38: 861-862.

19. Raglin JS (2001). Psychological factors in sport performance: the mental health model revisited. Sports Med 31(12): 875-890.

20. Rascle O, Coulomb G, Pfister R (1998). Aggression and goal orientation in handball: influence of institutional sport context. Percept Mot Skills 86: 1347-1360.

21. Reilly T, Williams AM, Nevill A (2000). A multidisciplinary approach to talent identification in soccer. $J$ Sports Sci
18(9): 695-702.

22. Saint-Phard D, Van Dorsten B, Marx RG, York KA (1999). Self-perception in elite collegiate female gymnastics, crosscountry runners and track-and-field athletes. Mayo Clin Proc 74: 770-74.

23. Samulski DM (2002). Psicologia do esporte: teoria e aplicação prática. Barueri: Manole.

24. Simpson S, Newby RW (1991). Personality profile of no scholarship college football players. Percept Mot Skills 73: 1083-1089.

25. Solomon GB (2001). Performance and personality impression cues as predictors of athletic performance: an extension of expectancy theory. Int J Sports Psychol 32(1): 88-100.

26. Thomas JR, Nelson JK (1996). Research Methods in Physical Activity. Champaign: Human Kinetics.

27. Vealey RS (1992) Personality and Sport: a comprehensive view. In: TS Horn (Ed). Advances in Sport Psychology. Champaign: Human Kinetics, 26-60.

28. Zapletalova L, Medekova H (2003). The relationship between children's personal traits, motor activity and motor performance. Gymnica 33(1):11-18.

29. Weinberg RS, Gould D (1995). Foundations of Sport and Exercise Psychology. Champaign: Human Kinetics.

30. Williams AM, Reilly T (2000). Talent identification in soccer. J Sports Sci 18: 657-667. 